\title{
Sets of Semi-Commutative Matrices
}

\author{
By J. Willtamson, Johns Hopkins University.
}

(Received 7th July, 1932. Received in revised form 8th November, 1932. Read 4th November, 1932.)

Introduction. In a paper entitled "Sets of anticommuting matrices" Eddington ${ }^{1}$ proved that if $E_{1}, E_{2}, \ldots, E_{2}$ form a set of $q$ four-rowed square matrices satisfying the relations,

$$
E_{i} E_{j}=-E_{j} E_{i}, \quad E_{i}^{2}=-E, \quad i, j=1,2, \ldots, q, \quad i \neq j,
$$

where $E$ is the unit matrix, then the maximum value of $q$ is five. Later Newman ${ }^{2}$ showed that this result is a particular case of the general theorem that if $E_{1}, E_{2}, \ldots, E_{u}$ form a set of $q$ t-rowed square matrices satisfying (1), where $t=2^{p} \tau$ and $\tau$ is odd, then the maximum value of $q$ is $2 p+1$.

In this paper we consider a generalization of Newman's theorem and prove the following result.

Theorem I. If $\omega$ is a primitive nth root of unity, and if $E_{1}, E_{2}, \ldots, E_{q}$. form a set of $q$ t-rowed square matrices satisfying the relations

$$
E_{i} E_{j}=\omega E_{j} E_{i}, \quad E_{i}^{n}=E, \quad i, j=1,2, \ldots, q, \quad i<j,
$$

where $E$ is the unit matrix and $t=n^{p} \tau, \tau \neq 0$ mod $n$, then the maximum value of $q$ is $2 p+1$. Moreover, for every value of $t$, sets of $2 p+1$ matrices satisfying (2) exist.

We shall call a set of $q$ matrices satisfying (2) an $E$-set; or in the case where $q$ is maximal, a maximal $E$-set. While Eddington and Newman proved a theorem on the number of real matrices in a maximal $E$-set for the case $n=2$, we shall see that no such theorem is true in the general case. However, if $n$ is even, there does exist a general theorem on the number of matrices of a special type in a maximal $E$-set.

[As a consequence of this it may be shown that, when $t=n^{p}$, every matrix of order $t$ can be expressed as a polynomial, with complex number coefficients, in the matrices of any maximal $E$-set. It is

I Journal London Math. Soc., 7 (1932), 58-68.

2 Ibid, 7 (1932), 94-99. 
also shown that any two maximal $E$-sets are similar, provided that $p \neq 0$. That is, if $F_{1}, F_{2}, \ldots, F_{q}$ form a set of matrices which satisfy the equivalent of (2), then there exists a non-singular matrix $A$ such that $A E_{i} A^{-1}=F_{i}, i=1,2, \ldots, q$. It is also possible to relate the $E$-sets of matrices of order $t$ with periodic collineations in space of $t-1$ dimensions, and thereby to obtain the different types of such maximal groups.]

$\S 1$. For the proof of Theorem I we require two lemmas.

Lemma 1. If $t=\tau \neq 0 \bmod n$, then the maximal number of matrices in an $E$-set is one.

For, if the set contains at least two members $E_{1}$ and $E_{2}$ such that $E_{1} E_{2}=\omega E_{2} E_{1}$, by taking the determinants of both sides of this last matrix equation we obtain

$$
\left|E_{1}\right|\left|E_{2}\right|=\omega^{t}\left|E_{2}\right|\left|E_{1}\right| ;
$$

and, since by (2) both $E_{1}$ and $E_{2}$ are non-singular, $\omega^{t}$ must be equal to unity. This result contradicts the fact that $t$ is not a multiple of $n$; and accordingly the lemma is proved. It is worth noticing that, since two matrices, which are both $n$th roots of the unit matrix, are not necessarily similar, so in the present case, if $t=\tau \neq 0 \bmod n$, two maximal $E$-sets, since each consists of a single member, are not necessarily similar.

LeMma 2. If $E_{1}$ is a member of an $E$-set, where $q>1$ and $t=k n$, then there exists a non-singular matrix $A$ such that

$$
A^{-1} E_{1} A=F_{1} \text {, where } F_{1}=\left[\begin{array}{cccccc}
e & 0 & 0 & . & . & 0 \\
0 & \omega e & 0 & . & . & 0 \\
. & . & . & . & . & . \\
. & . & . & . & . & . \\
0 & 0 & 0 & . & . & \omega^{n-1} e
\end{array}\right]
$$

and $e, w e, \ldots, \omega^{n-1} e$ are all scalar matrices of order $k, e$ being the unit matrix of order $k$.

For, since $E_{1}$ satisfies the characteristic equation $E_{1}^{n}-E=0$, the latent roots of $E_{1}$ are all powers of $\omega$, and there must also exist a non-singular matrix $B$ such that

$$
B E_{1} B^{-1}=G_{1},
$$

where $G_{1}$ is a diagonal matrix having these powers of $\omega$ in the 
diagonal. Let the latent root $\omega^{i}, j=0,1, \ldots, n-1$, appear exactly $t_{j}$ times in $G_{1}$ so that we have the equality

$$
k n=\sum_{j=0}^{n-1} t_{j}
$$

Now, if $E_{2}$ is a second non-singular matrix such that

then we have the result

$$
E_{1} E_{2}=\omega E_{2} E_{1}
$$

$$
E_{2}^{-1} E_{1} E_{2}=\omega E_{1} \text {. }
$$

Accordingly the latent roots of $E_{1}$ are the same as the latent roots of $\omega E_{1}$; and, as the latent roots of $\omega E_{1}$ are $\omega$ times the latent roots of $E_{1}$, multiplication by $\omega$ merely permutes the latent roots of $E_{1}$ amongst themselves. Now, if $\omega^{8}$ is the latent root of $E_{1}$, for which

$$
t_{s}=t \geqq t_{j}, \quad j=0,1,2, \ldots, n-1,
$$

then $\omega^{s+1}$ appears at least $t$ times amongst the latent roots of $\omega E_{1}$, and therefore at least $t$ times amongst the latent roots of $E_{1}$. Hence $t_{s+1} \geqq t$; and so, by $(6), t_{s+1}=t$. Similarly we can show that

$$
t_{s}=t_{s+1}=\ldots=t_{s+n-1}=t,
$$

where the subscripts must be reduced modulo $n$. From (5) it follows that $k=t$ and hence that (3) is true. An alternative statement of this lemma is as follows. The latent roots of any matrix of an $E$-set, which consists of more than one member, are the roots of unity $1, \omega, \omega^{2}, \ldots, \omega^{n-1}$, each repeated the same number of times.

It will now be shown by actual examples that matrices of the type postulated in Theorem I exist. If $t=n$, it may be verified without difficulty that the following three matrices satisfy (2):

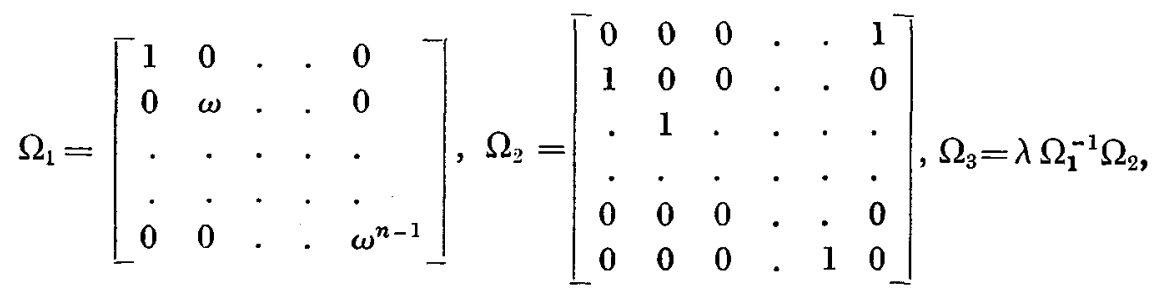

where $\lambda=1$, if $n$ is odd, and $\lambda=\sqrt{ } \bar{\omega}$, if $n$ is even. Further, if $E_{1}, E_{2}, \ldots, E_{f}$ form a set of $f$ matrices of order $m$ satisfying (2), then the matrices

$$
E_{1} \cdot \Omega, \quad E_{2} \cdot \Omega, \ldots, E_{f-1} \cdot \Omega, \quad E_{f} \cdot \Omega_{1}, \quad E_{f} \cdot \Omega_{2}, \quad E_{f} \cdot \Omega_{3},
$$

where $\cdot$ denotes direct product ${ }^{1}$ and $\Omega$ is the unit matrix of order $n$,

1 See L. E. Dickson, Algebras and their Arithmetics, p. 72. 
form a set of $f+2$ matrices of order $m n$ satisfying (2). For

$$
\begin{aligned}
& \begin{array}{l}
\left(E_{i} \Omega\right)^{n}=E_{i}^{n} \cdot \Omega^{n}=E, \quad i=1,2, \ldots, f-1 ; \\
\left(E_{f} \cdot \Omega_{j}\right)^{n}=E_{f}^{n} \cdot \Omega_{j}^{n}=E, \quad j=1,2,3 ; \\
\left(E_{i} \cdot \Omega\right)\left(E_{j} \cdot \Omega\right)=E_{i} E_{j} \cdot \Omega^{2}=\omega E_{j} E_{i} \cdot \Omega^{2}=\omega\left(E_{j} \cdot \Omega\right)\left(E_{i} \cdot \Omega\right), \\
\quad i<j ; i, j=1,2, \ldots, f-1 ; \\
\left(E_{i} \cdot \Omega\right)\left(E_{f} \cdot \Omega_{j}\right)=E_{i} E_{f} \cdot \Omega \Omega_{j}=\omega E_{f} E_{i} \cdot \Omega_{j} \Omega, \\
=\omega\left(E_{f} \cdot \Omega_{j}\right)\left(E_{i} \cdot \Omega\right), \quad i=1,2, \ldots, f-1 ; j=1,2,3 ; \\
\left(E_{f} \cdot \Omega_{i}\right)\left(E_{f} \cdot \Omega_{j}\right)=E_{f}^{2} \cdot \Omega_{i} \Omega_{j}=\omega E_{f}^{2} \cdot \Omega_{j} \Omega_{i} \\
=\omega\left(E_{f} \cdot \Omega_{j}\right)\left(E_{f} \cdot \Omega_{i}\right), \quad i<j ; i, j=1,2,3 .
\end{array}
\end{aligned}
$$

Thus, if there exists an $E$-set of matrices of order $m$ containing $f$ members, there exists an $E$-set of matrices of order $m n$ containing $f+2$ members. But, by Lemma I, there exists an $E$-set of matrices of order $r \neq 0 \bmod n$ containing one member; therefore, by induction there exists an $E$-set of matrices of order $t=n^{p} r$ containing $2 p+1$ matrices. This proves the last part of Theorem $I$.

If $m=k n$, by Lemma 2 there exists a non-singular matrix $A$ such that $A^{-1} E_{i} A=F_{i}$, where $F_{1}$ is given by (3). The matrices $F_{i}$ so defined also form an $E$-set; and, if we write $F_{s}=\left(f_{i j}\right), i, j=1,2, \ldots, n$, where each $f_{i j}$ is a matrix of order $k$, then since $F_{1} F_{s}=\omega F_{s} F_{1}$, we have $f_{i j}\left(\omega^{i-1}-\omega^{j}\right)=0$, or $f_{i j}=0$, if $i \neq j+1 \bmod n$. Accordingly $F_{s}$ has the form

$$
\left[\begin{array}{lllllll}
0 & 0 & . & . & . & 0 & F_{1 s} \\
F_{2 s} & 0 & . & . & . & 0 & 0 \\
0 & F_{3 s} & . & . & . & 0 & 0 \\
. & . & . & . & . & . & . \\
. & . & . & . & . & . & . \\
0 & 0 & . & . & . & F_{n s} & 0
\end{array}\right],
$$

where each $F_{i s}$ is a matrix of order $k$, and 0 denotes the zero matrix of order $k$. Since $F_{s}^{n}=E$, it follows that

$$
F_{n s} F_{n-1, s} \ldots F_{2 s} F_{1 s}=F_{1 s} F_{n s} \ldots F_{2 s}=\ldots=F_{n-1, s} \ldots F_{1 s} F_{n s}=e,
$$

and, if $s<u$, since $F_{s} F_{v}=\omega F_{u} F_{s}$, that

$$
F_{i s} F_{i-1, u}=\omega F_{i u} F_{i-1, s},
$$

where $F_{0 u}=F_{u u}$. But by (10) $F_{12}$ is non-singular and so the matrices

$$
G_{s}=\lambda F_{12}^{-1} F_{1 s} \text {, }
$$


of order $k$, exist for $s=3,4, \ldots, f$. We now proceed to show that, if $\lambda=1$ when $n$ is odd, and $\lambda=\sqrt{ } \bar{\omega}$ when $n$ is even, the matrices $G_{A}$ form an $E$-set containing $f-2$ members. For we have

Similarly

$$
\begin{aligned}
G_{d} G_{u} & =\lambda F_{12}^{-1} F_{1 s} \lambda F_{1:-1}^{-1} F_{1 u}, \\
& =\lambda^{2} F_{i 22} F_{n-1,2} \ldots F_{22} F_{1}, F_{n 2} \ldots F_{22} F_{1 u} \quad \text { by }(10), \\
& =\lambda^{2} \omega F_{n 2} F_{n-1,2} \ldots F_{32} F_{2 s} F_{12} F_{n 2} \ldots F_{22} F_{1 u} \quad \text { by }(11), \\
& =\lambda^{2} \omega F_{n 2} F_{n-1,2} \ldots F_{32} F_{2 s} F_{1 u} \quad \text { by }(10) .
\end{aligned}
$$

$$
G_{u} G_{s}=\lambda^{2} \omega F_{n 2} F_{n-1,2} \ldots F_{32} F_{2 u} F_{1 s}
$$

and, since $\quad F_{2 s} F_{1 u}=\omega F_{2 u} F_{1 s}$, we obtain $G_{s} G_{u}=\omega G_{u} G_{s}$.

Moreover

$$
\begin{aligned}
G_{s}^{n} & =\lambda^{n} F_{n 2} \ldots F_{22} F_{1 s}\left(F_{12}^{-1} F_{1 s}\right)^{n-1} \quad \text { by }(10), \\
& =\lambda^{n} \omega^{n-1} F_{n s}^{\prime} F_{n-1,2} \ldots F_{92} F_{12}\left(F_{12}^{-1} F_{1 s}\right)^{n-1} \quad \text { by }(\mathbf{1} \mathbf{1}), \\
& =\lambda^{n} \omega^{n-1} F_{n s} F_{n-1,2} \ldots F_{22} F_{1 s}\left(F_{12}^{-1} F_{1 s}\right)^{n-2} \\
& =\lambda^{n} \omega^{n-1+n-2} F_{n s} F_{n-1,8} F_{n-2,2} \ldots F_{22} F_{12}\left(F_{12}^{-1} F_{1 s}\right)^{n-2}
\end{aligned}
$$

By repeating this process $n-1$ times we finally arrive at the result that

$$
G_{s}^{n}=\lambda^{n} \omega^{d} F_{n s} F_{n-1, s} \ldots F_{28} F_{18}=\lambda^{n} \omega^{d} e,
$$

where $d=n-1+n-2+\ldots+2+1=n(n-1) / 2$. If $n$ is odd, $\omega^{d}=1$, while if $n$ is even, $\omega^{d}=\omega^{n / 2}$. In either case $\lambda^{n} \omega^{d}=1$. Hence, if there exists an $E$-set of matrices of order kn containing $f$ members, there also exists an $\dot{E}$-set of matrices of order $k$ containing $f-2$ members. Thus, if there existed an $E$-set containing more than $2 p+1$ matrices of order $t=2^{2} r, r \neq 0 \bmod n$, there would exist more than one member of an $E$-set of matrices of order $r$. But by Lemma I this last result is impossible and so Theorem $I$ is proved.

Now let $R(\omega)$ denote the field obtained by adjoining $\omega$ to the field of all rational numbers. Then, if $n$ is even, $\sqrt{ } \omega$ does not belong to the field $R(\omega)$ and so there exist at least two distinct types of

If $\omega$ is a primitive $n$th root of unity, $\sqrt{\omega}$ is a prinitive $2 n$th root of unity. A primitive $n$th root of unity satisfies an equation of degree $\phi(n)$, irreducible in the field of rational numbers, where $\phi(n)$ is the Euler $\phi$-function. If $n=2^{s} k$, where $k$ is odd, $\phi(n)=2^{r-1} \phi(k)$ and $\phi(2 n)=2^{s} \phi(k)$. Thus the degrees of the irreducible equations satisfied by $\omega$ and $\sqrt{\omega}$ are different. Hence the fields $R(\omega)$ and $R(\sqrt{\omega})$ cannot coincide. This is no longer true if $n$ is odd, since, if $n=2 f+1, \sqrt{\omega}=\omega^{f+1}$. 
matrices, $R$-matrices and $I$-matrices, which are defined in the following manner. A matrix is said to be an $R$-matrix, when each element of the matrix lies in the field $R(\omega)$; a matrix is said to be an I-matrix, when each element of the matrix is a product of a number of $R(\omega)$ and $\sqrt{ } \omega$. We shall now consider $E$-sets, whose members are either $R$-matrices or else $I$-matrices, and shall accordingly assume $n$ to be even. That this restriction does not lead to a triviality is apparent from the consideration of the matrices (7), of which two are $R$-matrices and one is an $I$-matrix. Further, if the number of $R$-matrices and the number of $I$-matrices in one $E$-set are equal respectively to the number of $R$-matrices and the number of $I$-matrices in a second $E$-set, we shall call the two $E$-sets $R$-congruent. Similarly if a matrix $A$ is an $R$-matrix or an $I$-matrix, according as a matrix $B$ is an $R$-matrix or an $I$-matrix, we shall call the two matrices $R$-congruent.

In order to determine the number of $R$-matrices and $I$-matrices, which may occur in a maximal $E$-set, we require two Lemmas.

Lemma 3. If the matrices $E_{i}, i=1,2, \ldots, f$, form an $E$-set, $(E)$, all of whose members are either $R$-matrices or else $I$-matrices, and if $r_{1}, r_{2}, \ldots, r_{t}$ are $t$ integers such that $1 \leqq r_{1}<r_{2}<\ldots<r_{t} \leqq f$, then there exists an $E$-set $R$-congruent to $(E)$, whose first $t$ members are the matrices $E_{r_{1}}, E_{r_{2}}, \ldots E_{r_{t}}$.

It is easily verified that the set of matrices $(T)$, where

$$
T_{i}=E_{i}, \quad i \neq j \text { or } j-1, \quad T_{j-1}=E_{j}, \quad T_{j}=E_{j-1}^{-1} E_{j}^{2},
$$

form an $E$-set. But, since the matrices $E_{j-1}$ and $T_{j}$ are $R$-congruent, the sets $(E)$ and $(T)$ are also $R$-congruent. In the same manner, if $j$ is replaced by $j-1$, from $(T)$ a set $(S)$ can be formed such that

$$
S_{j-2}=T_{j-1}=E_{j}
$$

and such that the sets $(E)$ and $(S)$ are $R$-congruent. By repeating this process $j-1$ times we finally arrive at a set $(K), R$-congruent to $(E)$, and such that its first member is $E_{j}$ and its $k$ th member is $E_{k}$, if $k>j$. If $j=r_{1}$, the set $(K)$ has for its first member $E_{r_{1}}$ and for its $r_{i}{ }^{\text {'h }}$ member $E_{r_{i}}, i>1$. By applying the same process, with $j=r_{2}$, $r_{2}-2$ times to the set $(K)$, we obtain a set $(P), R$-congruent to $(E)$, which has for its first two members $E_{r_{1}}$ and $E_{r_{2}}$. Finally in $r_{1}-1+r_{2}-2+\ldots+r_{t}-t$ steps we arrive at an $E$-set $R$-congruent to $(E)$, whose first $t$ members are the matrices $E_{r i}, i=1,2, \ldots t$, and so the lemma is proved. 
Lemma 4. If in an $E$-set consisting of $f$ matrices of order $t=k n, g$ of the members are $R$-matrices and $h=f-g$ are I-matrices and both $g$ and $h$ are different from zero, then there exists an $E$-set of matrices of order $k$, of which $g-1$ are $R$-matrices and $h-1$ are $I$-matrices.

Since in an $E$-set, satisfying the above hypotheses, at least one matrix is an $R$-matrix and at least one an $I$-matrix, there exists, by Lemma 3 , an $E$-set, $E_{i}, i=1,2, \ldots f$, which is $R$-congruent to the original set, and such that $E_{1}$ is an $R$-matrix and $E_{2}$ an $I$-matrix. Now, if $A$ is an $R$-matrix, the set $F_{i}=A^{-1} E_{i} A, i=1,2, \ldots f$, and the set $E_{i}$ are $R$-congruent. But, since $E_{1}$ is an $R$-matrix, the matrix $A$ in (3) must be an $R$-matrix, so that the set $F_{i}=A^{-1} E_{i} A$, where $F_{1}$ is defined by (3) and $F_{i}, i>1$, by (9), is $R$-congruent to the set $E_{i}$. As $E_{2}$ is an $I$-matrix, so is $F_{2}$, and accordingly $F_{12}$, being a sub-matrix of $F_{2}$, is also an $I$-matrix. Hence the set of matrices $G_{s}, s=3,4, \ldots f$, defined by (11), since $\lambda$ now has the value $\sqrt{\omega}$, and the set $F_{s}, s=3,4, \ldots f$, are $R$-congruent. But the set $F_{s}$ and the set $E_{g}, s=3,4, \ldots f$, are $R$-congruent and so the set $G_{s}$ and the set $E_{R}$ are $R$-congruent. Since the set $E_{s}, s=3,4, \ldots, f$ contains exactly $(g-1) R$-matrices and exactly $(h-1) I$-matrices, the lemma is proved.

We have already proved in Theorem I that maximal $E$-sets of matrices of order $t=n^{p} r$, where $r$ is not divisible by $n$, exist and that the number of matrices in such a set is $2 p+1$. We now suppose that the number of $R$-matrices in such a maximal $E$-set has one of the values

$$
\begin{aligned}
\text { (i) } & p-1 \text {; } \\
\text { (ii) } & p \\
\text { (iii) } & p+1 \text {; } \\
\text { (iv) } & p+2 \text {; }
\end{aligned}
$$

and proceed to show that in some cases we are led to a contradiction.

By repeated applications of Lemma 4 we deduce the existence of $E$-sets consisting respectively of the following matrices:

(i) three $I$-matrices of order $n r$;

(ii) one $I$-matrix of order $r$;

(iii) one $R$-matrix of order $r$;

(iv) three $R$-matrices of order $n r$.

But an $E$-set of matrices of order $n r$ contains the three members $E_{1}, E_{2}, E_{3}$, where as in (7), since $n$ is even, $E_{3}=\sqrt{\omega} E_{1}^{-1} E_{2}$, so that 
$E_{1}, E_{2}, E_{3}$ cannot all be $R$-matrices or all $I$-matrices. Moreover when $r$ is odd, an $I$-matrix of order $r$ cannot be a member of an $E$-set, for the determinant of an $I$-matrix of order $r$ is of the form $\sqrt{\omega} k$, where $k$ is a number of $R(\omega)$, while the determinant of a member of an $E$-set, being the product of $n$th roots of unity, must lie in $R(\omega)$. If, however, $r$ is even, a matrix of order $r$ which is a member of an $E$-set may be an $I$-matrix; for the matrix

$$
H=\sqrt{\omega}\left(h_{i j}\right), \quad i, j=1,2, \ldots \tau,
$$

where

$$
h_{i j}=0 \text {, if } j \neq i+1 \bmod n \text {; }
$$

$$
h_{i, i+1}=\omega^{-1}, i=1,2, \ldots, r / 2 ; h_{i, i+1}=1, i=r / 2+1, \ldots, r,
$$

is an $I$-matrix and, since $H^{\prime \prime}=E$, it is a member of an $E$-set. As the unit matrix of order $r$ is an $R$-matrix and at the same time a member of an $E$-set, whether $r$ is even or odd, we have shown that of the four possibilities (i), (ii), (iii), (iv) only (ii) and (iii) may occur, when $r$ is even, and (iii) alone, when $r$ is odd. Further by repeated applications of (8) we see that maximal $E$-sets of matrices, of order $t=n^{p} r$, $r$ not divisible by $n$, exist, in which the number of $R$-matrices is $p$ or $p+1$ when $r$ is even, and $p+1$ when $r$ is odd.

We are now in a position to prove the following theorem.

TheOREM 2. If in a maximal $E$-set of matrices, of order $t=n^{p} r, r \neq 0$ mod $n$, the members are restricted to be either $R$-matrices or else I-matrices, then the number of $R$-matrices in the set is $u$, where $u$ satisfies

$$
0 \leqq u \leqq 2 p+1, \quad u \equiv p+1 \bmod 4,
$$

or

$$
0 \leqq u \leqq 2 p+1, \quad u \equiv p+1 \text { or } p \bmod 4,
$$

according as $r$ is odd or even. Sets exist for every admissible value of $u$.

Let $E_{1}, E_{2}, E_{3}, \ldots, E_{q}=E_{2 p_{+1}}$ be a set $(E)$ of matrices of order $t$ satisfying the hypotheses of the theorem, and let $g$ of the matrices be $R$-matrices, and $h=2 p+1-g$ be $I$-matrices. Then the matrices in the $\operatorname{set}(F)$, defined by

$$
\left\{\begin{array}{l}
F_{i}=E_{i}, \quad 1 \leqq i<2 k, \\
F_{i}=\mu E_{1}^{-1} E_{2} E_{3}^{-1} E_{4} \ldots E_{2 k-1}^{-1} E_{i}=S_{k} E_{i}, \quad 2 k \leqq i \leqq q,
\end{array}\right.
$$

where $\mu=1$, if $k$ is even, and $\mu=\sqrt{\omega}$, if $k$ is odd, form an $E$-set for all values of $k$, where $\mathbf{l} \leqq k \leqq p$. For it is easily verified that $E_{i} S_{k}=S_{k} E_{i}$, if $i<2 k$, and that $E_{i} S_{k}=\omega S_{k} E_{i}$, if $i>2 k$. 
Accordingly

and

$$
\begin{aligned}
F_{i} F_{j} & =E_{i} E_{j}=\omega E_{j} E_{i}=\omega F_{;} F_{i}, \quad i<j ; i, j=1,2, \ldots, 2 k-1 ; \\
F_{i} F_{j} & =E_{\mathrm{i}} S_{k} E_{j}=S_{k} E_{i} E_{j}=\omega S_{k} E_{j} E_{i}=\omega F_{j} F_{i}, \quad i<2 k, j \geqq 2 k ; \\
F_{i} F_{j} & =S_{k} E_{i} S_{k} E_{j}=\omega S_{k}^{2} E_{i} E_{j}=\omega^{2} S_{k}^{2} E_{j} E_{i}=\omega S_{k} E_{j} S_{k} E_{i} ; \\
& =\omega F_{j} F_{i}, \quad i, j \geqq 2 k, i<j ;
\end{aligned}
$$

$$
\begin{aligned}
F_{i}^{n} & =E_{i}^{n}=E, \quad i<2 k ; \\
F_{i}^{n} & =\left(S_{k} E_{i}\right)^{n}=\mu^{n}\left(E_{1}^{-1} E_{2}\right)^{n}\left(E_{3}^{-1} E_{4}\right)^{n} \ldots\left(E_{2 k-1}^{-1} E_{i}\right)^{n}, \\
& =\mu^{n} \omega^{d} E, \quad d=-k n(n-1) / 2, \quad i \geqq 2 k, \\
& =E,
\end{aligned}
$$

if $\mu=1$, when $k$ is even, and $\sqrt{\omega}$, when $k$ is odd. But if $k$ is odd and the matrices $E_{1}, E_{2}, \ldots, E_{2 \bar{k}_{-1}}$ are all $R$-matrices, then the matrix $S_{k}$, defined by (15), is an $I$-matrix and the number of $I$-matrices in the set $F_{i}$ is

$$
v=g-(2 k-1) \equiv g-1, \bmod 4 .
$$

If $1 \leqq 2 k-1 \leqq g$ we may assume that the matrices $E_{1}, E_{2}, \ldots E_{2 k-1}$ are $R$-matrices, since otherwise, by Lemma (3), we can find a set $\left(E^{\prime}\right)$, $R$-congruent to the set $(E)$, of which the first $2 k-1$ members are $R$-matrices and so we can use the set $\left(E^{\prime}\right)$ instead of the set $(E)$ to define the set $(F)$. Thus $E$-sets exist, in which the number of $I$-matrices is $v$ for all values of $v$ satisfying

$$
v \equiv g-1, \bmod 4, \quad 0 \leqq v \leqq g-1 .
$$

On the other hand, if $k$ is odd, and $E_{1}, E_{2}, \ldots, E_{2 k-1}$ are all $I$-matrices, $S_{k}$ is still an $I$-matrix so that the number of $I$-matrices in the set $(F)$ is now

$$
v=2 k-1+g \equiv g-1, \bmod 4 .
$$

Once again, by Lemma 3 , for every value of $k, 3 \leqq 2 k-1 \leqq h$, we can find a set $\left(E^{\prime}\right), R$-congruent to $(E)$, such that its first $2 k-1$ members are $I$-matrices, and so there exist $E$-sets in which the number of $I$-matrices is $v$, for all values of $v$ satisfying

$$
v \equiv g-1, \bmod 4, \quad g+3 \leqslant v \leqslant 2 p+1 .
$$

Accordingly by (16) and (17), if an $E$-set of $2 p+1$ members exists, in which $g$ of the matrices are $R$-matrices, and $2 p-g+1$ are 
$I$-matrices, then there exists an $E$-set, in which the number of $I$-matrices is $v$, where $v$ satisfies

$$
v \equiv g-1, \bmod 4, \quad 0 \leqslant v \leqslant 2 p+1 .
$$

But, if $r$ is odd, $E$-sets exist in which $g=p+1$, while, if $r$ is even, $E$-sets exist in which $g$ is either $p$ or $p+1$. Accordingly $E$-sets do exist in which the number of $R$-matrices is $u$, for every value of $u$ satisfying (13) if $r$ is odd, and (14) if $r$ is even. If there existed an $E$-set in which the number $u$ of $R$-matrices did not satisfy (13) or (14), there would exist an $E$-set in which $u$ had the value $p$, $p-1$, or $p+2$, in the one case, and $p+2$ or $p-1$ in the other. As it has already been shown that such $E$-sets cannot exist, Theorem 2 is proved.

No such theorem is true when $n$ is odd, for, as already remarked, there is then no distinction between $I$-matrices and $R$-matrices. If $n=2$, we have $\omega=-1$ and $\sqrt{\omega}=i$, so that $R$-matrices are real rational matrices, while $I$-matrices are pure imaginary matrices. $R(\omega)$ is now the field of all rational numbers, but in this particular case the argument would remain unaltered if the field of all real numbers were used instead. Since, if $n=2, r$ must be odd, in Theorem 2 only formula (13) is required. ${ }^{1}$

1 As both Eddington and Newman consider matrices whose squares are $-E$, the number of imaginary matrices in a set of such matrices is the same as the number of real matrices in an $E$-set, satisfying (2) with $n=\mathbf{g}$. 